\title{
Secondary pulmonary alveolar proteinosis predominant in the transplanted lung in patients with idiopathic interstitial pneumonia: an autopsy case
}

\author{
Satoshi Ikeda ${ }^{1,2}$, Akimasa Sekine ${ }^{1}$, Tomohisa Baba ${ }^{1}$, Takuma Katano ${ }^{1}$, Hideaki Yamakawa ${ }^{1}$, Tsuneyuki \\ $\mathrm{Oda}^{1}$, Tae Iwasawa ${ }^{3}$, Mai Matsumura ${ }^{2}$, Tamiko Takemura ${ }^{4}$, Takashi Ogura ${ }^{1}$ \\ ${ }^{1}$ Department of Respiratory Medicine, Yokohama, Kanagawa Cardiovascular and Respiratory Center, Japan; ${ }^{2}$ Department of Pathology, Yokohama- \\ city University Graduate School of Medicine, Yokohama, Japan; ${ }^{3}$ Department of Radiology, Kanagawa Cardiovascular and Respiratory Center, \\ Yokohama, Japan; ${ }^{4}$ Department of Pathology, Japanese Red Cross Medical Center, Tokyo, Japan \\ Correspondence to: Satoshi Ikeda, MD. Department of Respiratory Medicine, Kanagawa Cardiovascular and Respiratory Center, Tomioka-Higashi \\ 6-16-1, Kanazawa-ku, Yokohama 236-0051, Japan. Email: isatoshi0112@gmail.com.
}

\begin{abstract}
A man in his $40 \mathrm{~s}$ with idiopathic interstitial pneumonia underwent cadaveric left singlelung transplantation from a brain-dead donor in October 2014. In October 2015, chest high-resolution computed tomography revealed centrilobular ground-glass opacities (GGOs) predominantly in the transplanted left lung, and subsequently, the shadows progressed to a geographic GGO without crazy paving. Bronchoalveolar lavage fluid analysis revealed an opaque and milky appearance, and cytopathology demonstrated foamy alveolar macrophages and abundant granular, acellular, eosinophilic, and amorphous material in the background. There was no evidence of infection. Serum anti-granulocyte-macrophage colony-stimulating factor antibody testing was negative. We diagnosed the patient with secondary pulmonary alveolar proteinosis (PAP) following lung transplantation. Autopsy revealed PAP findings predominant in the transplanted left lung, which also had dilated lymphatic vessels. In addition to defects in alveolar macrophage function from immunosuppressive therapy, impaired lymphatic drainage due to transplantation would contribute to the onset of secondary PAP in the transplanted lung.
\end{abstract}

Keywords: Pulmonary alveolar proteinosis (PAP); lung transplantation; complications

Submitted Dec 24, 2017. Accepted for publication Apr 06, 2018.

doi: $10.21037 /$ jtd.2018.04.49

View this article at: http://dx.doi.org/10.21037/jtd.2018.04.49

\section{Introduction}

Since 1983, more than 50,000 lung transplants have been performed worldwide (1). To date, few cases of secondary pulmonary alveolar proteinosis (PAP), as a rare complication following lung transplantation, have been reported (2-6) and the mechanism of pathogenesis is not fully clear.

\section{Case presentation}

A man in his $40 \mathrm{~s}$ was admitted to our hospital with complaints of dry cough and dyspnea on exertion in May 2008. Chest high-resolution computed tomography (HRCT) revealed peribronchovascular reticulation with lower lobe predominance. In September 2008, a surgical lung biopsy revealed a fibrosing nonspecific interstitial pneumonia pattern associated with a desquamative interstitial pneumonia-like reaction. In October 2008, prednisolone and cyclosporine were administered; however, the interstitial pneumonia gradually worsened. In October 2014, the patient underwent cadaveric left single-lung transplantation from a brain-dead donor. Mycophenolate mofetil was initiated for prevention of acute rejection. In October 2015, postoperative chest HRCT revealed centrilobular ground-glass opacities (GGOs) in the transplanted left lung (Figure 1A). The possibility of infection was eliminated based on sputum and blood culture results, serum $\beta-\mathrm{D}$ glucan and cytomegalovirus 



Figure 1 High-resolution computed tomography findings. Chronological changes of high-resolution computed tomography (HRCT) findings are presented. In October 2015, (A) postoperative chest HRCT revealed centrilobular ground-glass opacities (GGOs) in the transplanted left lung. In February 2016, (B) the shadow changed to a geographic GGO without crazy paving in the left lung.

antigen testing, and the ineffectiveness of antibacterial/ antifungal agents. Although methylprednisolone pulse therapy was administered due to lung transplant rejection signs such as lymphocytic bronchiolitis, imaging revealed no improvement. In February 2016, the shadow changed to a geographic GGO without crazy paving in the left lung (Figure 1B). Bronchoalveolar lavage fluid analysis revealed an opaque and milky appearance, and cytopathology demonstrated foamy alveolar macrophages and abundant granular, acellular, eosinophilic, amorphous, and periodic acid-Schiff-positive material in the background (Figure 2A), with no microorganisms. Thus, the patient was diagnosed with PAP. Serum anti-granulocyte-macrophage colonystimulating factor antibody testing was negative. As the patient lacked respiratory symptoms, whole lung lavage (WLL) was not performed and his condition was carefully monitored while adjusting the doses of immunosuppressants. However, beginning in late March 2016, his HRCT findings gradually worsened. At this time, the patient and her family did not choose to perform WLL because of the concerns over intubation and ventilator. Aspergillus terreus was cultured from sputum, and thus, voriconazole was initiated. However, he developed acute respiratory failure and died in April 2016. Autopsy revealed PAP findings predominant in the transplanted left lung, which also had dilated lymphatic vessels (Figure 2B,C,D). Cause of death was invasive pulmonary aspergillosis.

\section{Discussion}

PAP occurs in three distinct forms: autoimmune, genetic, and secondary (7). In the present case, serum antigranulocyte-macrophage colony-stimulating factor antibody test was negative. Although the possibility of genetic PAP cannot be completely excluded, no PAP was detected in the surgical lung biopsy specimen from the right lung in September 2008 or from the extracted left native lung in October 2014; PAP occurred after lung transplantation, predominantly in the transplanted lung. Thus, we diagnosed the patient with secondary PAP following lung 


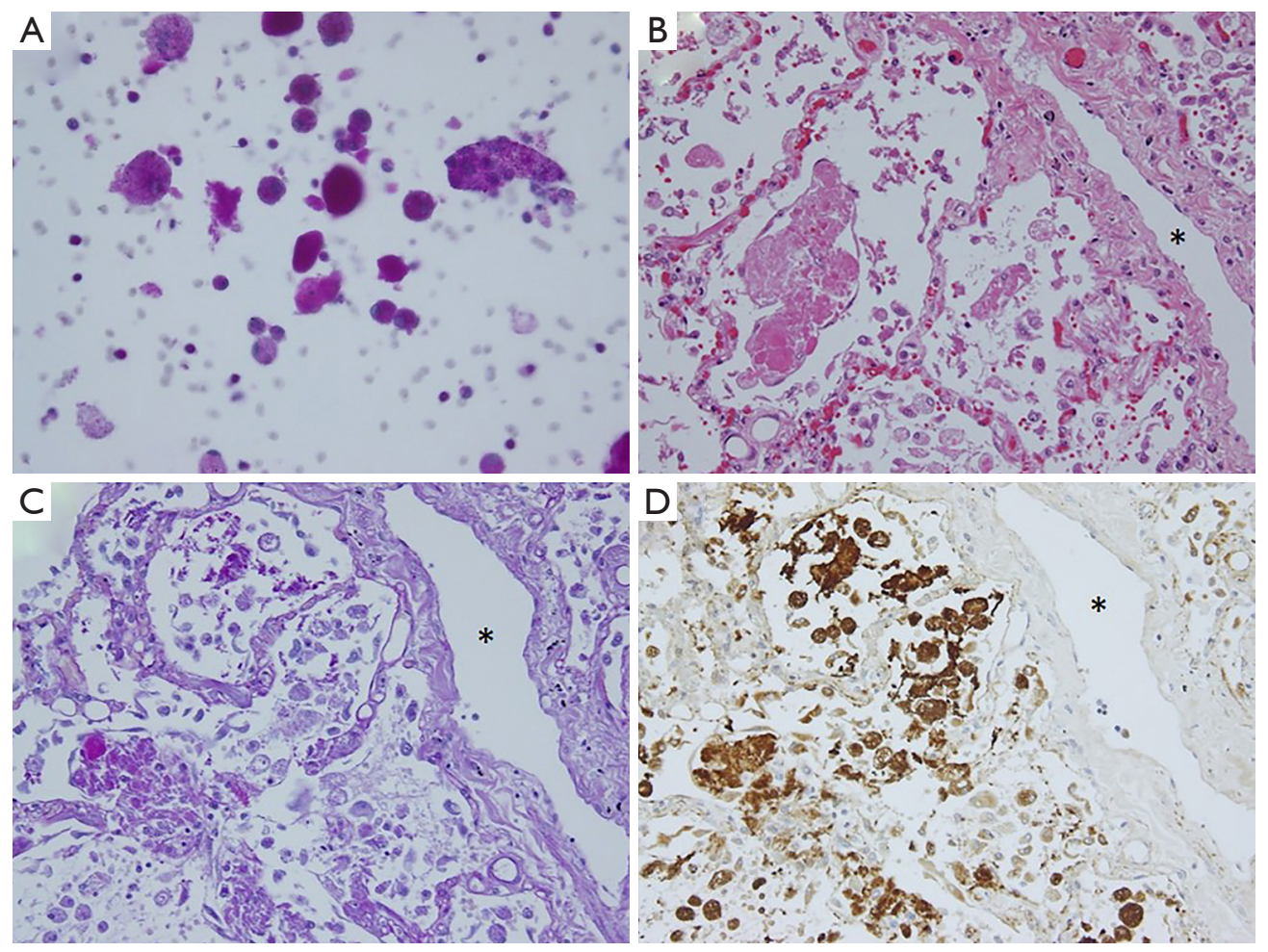

Figure 2 Representative photographs from microscopic examination. Microscopic appearance of the bronchoalveolar lavage (BAL) is shown in (A). (A) BAL fluid analysis revealed an opaque and milky appearance, and cytopathology demonstrated foamy alveolar macrophages and abundant granular, acellular, eosinophilic, amorphous, and periodic acid-Schiff (PAS)-positive material in the background (PAS stain, $\times 400)$. (B,C,D) microscopic appearance of the autopsied transplanted left lung. (B) Autopsy revealed intra-alveolar accumulation of granular, acellular, eosinophilic, and amorphous material and numerous alveolar macrophages, with a preserved alveolar septal architecture (hematoxylin and eosin stain, $\times 200$ ); (C) intra-alveolar materials were positive for the PAS stain (PAS and Alcian-blue stain, $\times 200)$ and (D) surfactant-protein A immunohistochemistry $(\times 200)$. In addition, lymphatic vessels were dilated in the transplanted left lung $\left(^{*}\right)$.

transplantation.

The present case demonstrated two important clinical observations. First, PAP occurred predominately in the transplanted lung. Second, newly appearing shadows progressed from centrilobular GGOs to a geographic GGO without crazy paving.

To date, the onset mechanism of secondary PAP has been reported to be defects in alveolar macrophage function from immunosuppressive therapy (4). Therefore, most reported cases of secondary PAP after doublelung transplantation revealed a bilateral distribution $(5,6)$. However, in the present case, PAP findings were predominant in the transplanted lung. Similarly, reported cases of secondary PAP after single-lung transplantation showed transplanted lung predominance $(4,6)$. Moreover, in the present case, autopsy revealed dilation of lymph vessels in the transplanted lung (Figure $2 B$ ), which would reflect the dysfunction of lymphatic drainage. In the lung transplantation procedure, the severed lymphatic vessels from the donor's lungs are not surgically reconnected to the recipient's lungs due to technical limitations (8). Therefore, impaired lymphatic drainage due to transplant would contribute to the onset of secondary PAP.

The present case showed chronological changes in the secondary PAP imaging findings. Diffuse GGOs without crazy paving are common HRCT findings in patients with secondary PAP (9), as with this case. Although centrilobular GGOs, as shown primarily in this case, are atypical findings for PAP, there was a report of secondary PAP following double-lung transplantation with centrilobular GGOs (5). These findings could represent an incipient presentation of secondary PAP. However, in the present case, we did not consider the possibility of PAP in the early stage. Furthermore, methylprednisolone pulse therapy, 
administered due to the possibility of lung transplant rejection, might have induced further dysfunction of alveolar macrophages and subsequently worsened PAP. Thus, when centrilobular GGOs develop after lung transplantation, especially in the transplanted lung, chest physicians should take secondary PAP into consideration as an important differential diagnosis and perform bronchoscopic examination as soon as possible.

Since secondary PAP after lung transplantation is probably related to alveolar macrophage dysfunction caused by immunosuppressants, reducing immunosuppressants and/or using alternative agents have been preferred over WLL. However, the patients with secondary PAP often showed subsequent lung infections, resulting in fatal outcome. Although there have been few experiences with the efficacy or safety of WLL in lung transplant recipients, the immunomodulatory effects are expected with WLL. Thus, chest physicians need to consider WLL shortly after diagnosis of secondary PAP and/or in case of infection even if considered as non-severe initially.

\section{Acknowledgements}

The authors would like to thank Koh Nakata (Bioscience Medical Research Center, Niigata University Medical and Dental Hospital, Niigata, Japan) for the measurement of anti-granulocyte-macrophage colony-stimulating factor antibody.

\section{Footnote}

Conflicts of Interest: The authors have no conflicts of interest to declare.

Informed Consent: Written informed consent was obtained for publication of this case report and any accompanying images.

\section{References}

1. Yusen RD, Edwards LB, Kucheryavaya AY, et al. The registry of the International Society for Heart and Lung Transplantation: thirty-second official adult lung and heart-lung transplantation report--2015; focus theme: early graft failure. J Heart Lung Transplant 2015;34:1264-77.

2. Yousem SA. Alveolar lipoproteinosis in lung allograft recipients. Hum Pathol 1997;28:1383-6.

3. Parker LA, Novotny DB. Recurrent alveolar proteinosis following double lung transplantation. Chest 1997;111:1457-8.

4. Albores J, Seki A, Fishbein MC, et al. A rare occurrence of pulmonary alveolar proteinosis after lung transplantation. Semin Respir Crit Care Med 2013;34:431-8.

5. Darley DR, Malouf MA, Glanville AR. A rare case of everolimus-induced pulmonary alveolar proteinosis. J Heart Lung Transplant 2016;35:147-8.

6. Philippot Q, Cazes A, Borie R, et al. Secondary pulmonary alveolar proteinosis after lung transplantation: a singlecentre series. Eur Respir J 2017;49:1601369.

7. Borie R, Danel C, Debray MP, et al. Pulmonary alveolar proteinosis. Eur Respir Rev 2011;20:98-107.

8. Cui Y, Liu K, Lamattina AM, et al. Lymphatic vessels: The next frontier in lung transplant. Ann Am Thorac Soc 2017;14:S226-32.

9. Ishii H, Trapnell BC, Tazawa R, et al. Comparative study of high-resolution CT findings between autoimmune and secondary pulmonary alveolar proteinosis. Chest 2009;136:1348-55.
Cite this article as: Ikeda S, Sekine A, Baba T, Katano T, Yamakawa H, Oda T, Iwasawa T, Matsumura M, Takemura T, Ogura T. Secondary pulmonary alveolar proteinosis predominant in the transplanted lung in patients with idiopathic interstitial pneumonia: an autopsy case. J Thorac Dis 2018;10(5):E351-E354. doi: 10.21037/jtd.2018.04.49 